XVII.

\title{
Ueber die Wirkungen des Physostigmins auf muskuläre Organe.
}

Mit Beziehung auf die Untersuchungen von Dr. H. Winterberg.

Von

Professor Dr. Erich Harnack (Halle a. S.).

Als ich vor mehr als dreissig Jahren gemeinsam mit Dr. Witkowski ${ }^{1}$ ) daran ging, die Wirkungen des Physostigmins möglichst allseitig zu analysiren, da waren wir genöthigt, uns zuvor das Alkaloid aus der Bohne rein darzustellen. Die damaligen Handelspräparate bestanden aus krümlichen schwarzbraunen Schmieren, und die meisten Forscher, die vor uns mit Physostigmin gearbeitet, hatten sich eben unreiner Präparate bedient. In der Augenheilkunde wurde nur das Calabarbohnenextract (in kleinen Papierquadraten!) angewendet. Die lsolirung des Alkaloides war wegen der Zersetzlichkeit seiner Lösungen keineswegs leicht, aber es gelang uns, dasselbe in Form einer klaren hellgelben Masse, die zu leimartigen Plättchen eintrocknete, zu gewinnen, indem wir im Wesentlichen einer von Hesse angegebenen Methode folgten. Irgend eine Verbindung der Base in krystallisirter Form herzustellen war uns nicht möglich, daher wir auf die Elementaranalyse verzichteten. Nach dem ganzen Verhalten erhielten wir den Eindruck, dass wir es mit einem sehr complicirt zusammengesetzten Moleküle zu thun hätten. Gleich darauf gelang es dann der Firma E. Merck in Darmstadt, das Salicylat der Base in Form einer klaren, scheinbar unkrystallisirten, schwach gelblichen Masse darzustellen. Dies ist das Präparat, welches ich nun etwa 28 Jahre aufbewahrt und Herrn Dr. Winterberg ${ }^{2}$ ) für seine Versuche überlassen habe, bei denen es sich als sehr stark wirksam erwies. Sicherlich ein seltenes Präparat, wenn man erwägt, dass das Physostigmin doch zu den ziemlich leicht zersetzlichen Alkaloiden gehört. Merck stellte dann aber bald auch ein schön krystallisirtes Salicylat des Physostigmins her, das ich ${ }^{3}$ ) in Betreff der miotischen Wirkung prüfte und sebr wirksam fand und das

1) Harnack und Witkowski, Mrchiv f. exper. Path. u. Pharmakol. Bd. V. S. 401.1876.

2) Winterberg, Diese Zeitschr. Bd. IV. 1907. 1.

3) Harnack, Archiv f. exper. Path. u. Pharmakol. Bd. XII. S. 336 f. 1880. 
daraufhin in den Arzneischatz aufgenommen wurde. Später haben französische Chemiker das Alkaloid analysirt und die Bruttoformel: $\mathrm{C}_{15} \mathrm{H}_{21}$ $\mathrm{N}_{3} \mathrm{O}_{2}$ gefunden. Wenn nun darnach auch der Stickstoffgehalt für ein Alkaloid ein selten hoher ist und über 15 pCt. beträgt, so muss ich doch gestehen, ich hatte eigentlich erwartet, dem Physostigmin würde ein höheres Molekulargewicht zukommen. $O b$ nun das Physostigmin in diesem schön krystallisirten Salze mit dem von uns damals dargestellten amorphen vollkommen identisch ist, das ist vorläufig noch nicht sicher anzugeben, freilich auch nicht das Gegentheil bewiesen. Winterberg fand das ihm von mir gelieferte, so alte Präparat sogar noch etwas kräftiger wirksam als das jetzige von Merck bezogene krystallisirte Salicylat! In der Art der Wirkung konnte er aber keinen Unterschied constatiren. Zu beachten ist auch, dass man später ein Is ophysostigmin aus der Bohne gewonnen hat, das dem Physostigmin isomer, aber als freie Base in Aether schwer löslich sein, dabei sehr energisch myotisch wirken soll. Die Frage bleibt also vorläufig eine offene, ich will daher nicht weiter daraul eingehen und mich auf den Standpunkt stellen, als ob die Präparate des wirklichen Physostigmins von damals und jetzt identisch seien.

Bei eingehender Analyse der Wirkung nach allen Richtungen hin gelangten Witkowski und ich zu dem Wahrscheinlichkeitsschlusse, dass das Physostigmin, was seine Wirkung auf Organe mit contractilen Elementen (Herz, Iris, Darm, willkürliche Muskeln) anlangt, auf die lebende glatte und quergestreifte Muskelfaser selbst einwirkt, indem es deren Contractilität erhöht oder direct als Reiz wirkt. In Betreff des M. sphineter iridis war übrigens diese Annahme schon vor uns von dem Franzosen Martin Damourette ausgesprochen worden. Von den späteren Forschern hat ein Theil unsere Angaben bestätigt und vervollständigt, während Andere, ohne das thatsächliche Material wesentlich zu vermehren, durch ihre Beobachtungen zu dem Schlusse gelangten, dass an den bezeichneten Wirkungsorten die betreffenden Nervenendap parate durch das Physostigmin gereizt werden, die Muskelfasern selbst aber nicht oder doch nicht in erster Linie. Es handelt sich also zunächst um die Deutung der übereinstimmend festgestellten Thatsachen, d. h. mit anderen Worten, um die genaue Localisirung der Wirkung. Auf die sich ausschliesslich auf das Warmblüterherz beziehenden nevesten Untersuchungen von Winterberg gehe ich unten näher ein. Ich möchte nun zunächst darauf hinweisen, auf Grund welcher Erwägung Witkowski und ich zu unserer Annahme, dass die Wirkung die Muskelfaser betrifft, gelangt sind. Es geschah auf Grund einer allgemeinen Voraussetzung, die damals wohl von nahezu allen Pharmakologen getheilt wurde. Man hielt es für unmöglich, dass ein Nervenapparat, der durch ein lähmendes Gift seiner Erregbarkeit vollständig beraubt worden, durch ein gleich darauf zugeführtes erregendes Gift überhaupt beeinflusst werden könnte, sofern eben das erstere Gift noch nicht eliminirt worden und die complet lähmende Wirkung demnach noch zu unterhalten im Stande wäre; denn - so meinte man - complet gelähmt heisst eben: zur Zeit durch nichts erregbar. Man nahm daher auch 
an, dass der erregend wirkende Antagonist, wenn er mit dem complet lähmenden genau auf den gleichen Nervenapparat einwirkte, gegen den letzteren niemals aufzukommen im Stande sei, während die umgekehrte Gegenwirkung sich leicht erzielen, der zuvor erregte Apparat sich ohne grosse Schwierigkeit lähmen lässt. Als Beispiel kann man etwa anführen das Verhalten des Apomorphins in der Narkose. Durch Chloroform etc. wird das Brecheentrum gelähmt, so dass in tiefer Narkose das Apomorphin nicht emetisch wirkt; das Respirationscentrum wird aber nicht gelähmt und kann in tiefer Narkose durch Apomorphin so heftig gereizt werden, dass die Frequenz der Athmung sich verachtfacht.

Die allgemeine Gültigkeit des obigen Satzes ist mir aber doch im Laufe der Zeiten allmählich zweifelhaft geworden. Was wissen wir denn überhaupt von den Bezichungen des einen oder des anderen Giftes zu dem Chemismus eines bestimmten Nervenapparates oder auch der lebenden Zelle überhaupt? Wenig oder nichts. Selbst die gegenwärtig mit besonderer Vorliebe und keineswegs erfolglos betriebene Forschung über den Zusammenhang zwischen chemischer Structur und Wirkungsart giebt uns über das eigentliche Warum? doch noch keinen Aufschluss, abgesehen davou, dass die Grundlage der ganzen Structurtheorie doch noch nicht als eine für alle Zeiten unabänderlich feststehende angesehen werden kann. Mit allen den schönen Vorstellungen, dass es in einem Fall das Fett, im anderen das Lecithin sei, welches in der Nervenzelle die Ursache für die chemisch-physikalische Anziehung zwischen letzterer und dem Agens bilde, ist doch noch wenig anzufangen. Ehrlich hat neuerdings gegen die pharmakologische Forschung den Vorwurf erboben, sie frage zu ausschliesslich nach dem Was und Wie, zu wenig nach dem Warum. Ich halte diesen Vorwurf für unbegründet; denn Untersuchụngen, die den zur Lösung der Frage Warum? führenden Weg bahnen, werden heut zu Tage, wie gesagt, in grosser Zahl und in weitem Umfang ausgeführt und haben für die Gewinnung "synthetischer Arzneimittel" schon werthvolle Früchte gezeitigt. Und wenn wir nach dem Warum? forschen, weshalb mit den complicirtesten Agentien beginnen, von deren Structur wir noch kein Ahnung haben, warum nicht mit den einfachsten, wie etwa Quecksilber, Blei etc., in deren Molekülen es keine haptophoren und toxiphoren Gruppen geben kann?

Wir wissen nur, dass der Nervenapparat oder die Zelle in dem einen Moment das ihnen nahe gebrachte Agens mit Begierde aufnimmt, um es nach einiger Zeit (falls nicht das Agens zerstört oder die Zelle vernichtet worden ist) mit der gleichen Energie wieder zu eliminiren. Wird nun der Zelle bald nach dem ersten Agens ein zweites zugeführt, wer kann wissen, ob sie letzteres - je nach seiner Qualität - nicht noch begieriger aufnimmt, dadurch aber in den Stand gesetzt wird, das erste um so rascher zu eliminiren oder zu vernichten? War das erste ein lähmendes Gift, so ist allerdings die specifische Function der Zelle zeitweilig sistirt, aber das braucht nicht zu verhindern, dass das zweite - erregende - Gift seinen Weg in die Zelle ete. findet und nun (ich 
habe dabei natürlich cinen Nervenapparat im Sinne) die Funetion wiederherstellen hilft.

Die Möglichkeit, dass auch bei einem wahren Antagonismus das erregende Gift Sieger bleiben kann, nicht muss, wird also nicht in Abrede gestellt werden können. Nimmt man diese Möglichkeit an, dann fällt allerdings der Zwang, die Physostigminwirkung als eine direct muskuläre aufzufassen, weg; nimmt man sie dagegen nicht an, dann bleibt freilich schwer für eine andere Auffassung Raum.

Beim Physostigmin liegt nämlich die Sache so, dass es am Herzen, der Iris, dem Darm, den willkürlichen Muskeln etc. über die die bezüglichen Nervenendapparate complet lähmenden Gifte Sieger bleibt. Hält man etwas Derartiges für den Fall eines wahren Antagonismus für unmöglich, dann bleibt nur die Annahme, dass das Physostigmin die Muskelfaser selbst becinflusst, oder man muss zu sehr complicirten Vorstellungen über die Einrichtung der Nervenendapparate greifen, indem man sich dieselben etwa in mehrere Strecken getheilt denkt, von denen die am periphersten gelegene zwar vom erregenden Physostigmin, nicht aber von den bezüglichen lähmenden Giften beeinflusst wird. Der Antagonismus wäre dann wieder kein "wahrer".

Auf die Thatsache, dass das Physostigmin über die lähmenden Gifte Sieger bleibt, möchte ich im Einzelnen etwas näher eingehen. Zuniichst in Bezug auf das Auge, die pupillenverengernde Wirkung. Hier erweist sich das Physostigmin als Sieger über das Atropin, was andere Myotica (Pilokarpin, Muskarin etc.) eben nicht vermögen, die zum Theil deswegen für die Augenheilkunde auch nicht die gleiche Brauchbarkeit besitzen als jenes. Bringt man in jedes Auge einer grossen Katze je einen Tropfen einer 1 proc. Atropinsulfatlösung, unter Verhütung jeden Verlustes, so zeigen sich nach gewisser Zeit beide Pupillen als maximal erweitert, ca. $12 \mathrm{~mm}$ im Durchmesser, die Irisränder bei Vorderansicht nicht mehr sichtbar. Bringt man nun z. B. ins linke Auge die gleiche Menge Physostigminsalicylat (2 gtt. einer $1 / 2$ proc. Lösung), so ist nach knapp zwei Stunden die rechte Pupille noch genau so weit wie vorher, die linke dagegen selbst bei trüber Beleuchtung auf $\mathbf{4 - 5} \mathrm{mm}$ im Querdurchmesser reducirt. Stellt man den Versuch so an, dass man gleichzeitig in das rechte Auge das Physostigmin, in das linke das Atropin (in den obigen gleichen Dosen) einträufelt, so sieht man nach zwei Stunden die rechte Pupille zu einem eben noch sichtbaren feinen Strich ${ }^{1}$ ) contrahirt, bei voller Beschattung sich vorübergehend mässig dilatirend. Dagegen ist die linke maximal erweitert, auf Belichtung nicht reagirend. Giebt man jetzt, und zwar wieder in den obigen gleichen Dosen, in das rechte Auge Atropin, in das linke Physostigmin, so beobachtet man

1) Bei der Physostigminmiose berühren sich wohl die Irisränder nie, was bei der örtlichen Muskarinwirkung sehr wohl der Fall sein kann. Auch dieses Moment scheint für gewisse Unterschiede in der Wirkung beider zu sprechen: vielleicht reizt das Physostigmin auch die - freilich bestrittenen - schwachen dilatatorischen Muskelfasern der Iris? 
nach oiner Stunde, dass beide Pupillen bei sohr schwacher Beleuchtung eine gleiche mittlere Weite $(8-9 \mathrm{~mm})$ bekommen haben, die zunächst auch anhält.

Bei Anwendung gleicher Dosen also contrahirt das Physostigmin die Atropinpupille auf ca. $1 / 3$ des Durehmessers (bei überwiegender Dosis des ersteren sogar noch mehr); umgekehrt wird die zuvor durch Physostigmin verengerte Pupille durch die gleich grosse Atropindosis zu einer mittleren, nicht maximalen Erweiterung gebracht. Es gewinnt also den Anschein, als ob immer das zuletzt zugeführte Agens der Sieger bliebe, und das liesse sich so deuten, dass bei der Aufnahme des zweiten Giftes das erste aus dem Nervenapparat theilweise verdrängt und eliminirt wird. Auffallend bleibt aber immerhin, dass z. B. das Muskarin sich anders verhält: es wirkt auf die Atropinpupille gar nicht, und die durch Muskarin verengerte Pupille wird durch Atropin maximal erweitert wie die normale. Nimmt man an, das Physostigmin reize den M. sphincter selbst, so erklären sich die Thatsachen scheinbar ganz gut; denn dass die Physostigminpupille durch Atropin zu einer mittleren Weite gebracht wird, ist begreiflich, da mit der Lähmung der Oculomotoriusendungen alle Reize allmählich wegfallen, die dem Sphineter vom Centrum her zugehen. Auch bei plötzlicher voller Beschattung pflegt sich die Physostigminpupille ein wenig zu erweitern, was sich aus dem plötzlichen Wegfall der auf den Sphincter durch Reflexact übertragenen Lichtreize erklären lässt. Den gleichen Effect muss selbstverständlich die Durchschneidung des N. oculomotorius ausüben.

$\Lambda \mathrm{m}$ Darme gestalten sich die Verhältnisse ganz analog, indem auch hier das Physostigmin über das die bezüglichen Nervenapparate in der Darmwand lähmende $\Lambda$ tropin Sieger bleibt und einen Darmkrampf auch nach der durch Atropin bewirkten Erschlaffung des Darmes hervorbringt. Auch hier vermag das Muskarin unter den gleichen Bedingungen nicht das Gleiche zu bewirken. Die Alternative bleibt demnach die gleiche: entweder Wirkung des Physostigmins auf die Muskelfaser selbst, oder das lähmende Gift - wird durch das erregende Physostigmin (nicht aber durch Muskarin) aus den Nervenapparaten gleichsam verdrängt, resp. seine Eliminirung befördert. Die Darmwirkung ist es auch nahezu allein, vermöge deren das Physostigmin ausserhalb der Augenheilkunde heut zu Tage noch praktisch benutzt wird. Viele rühmen seine Wirksamkeit bei Darmträgheit und -atonie in hohem Grade; immerhin ist - zumal bei der üblichen subcutanen Anwendung - Vorsicht wogen der lebensgefährlichen Wirkungen des Physostigmins auf Rückenmark, Medulla etc. geboten.

Ganz ähnlich liegen auch die Verhältnisse an den willkürlichen Muskeln: nur ist es hier nicht das Atropin, sondern das die motorischen Nervenendapparate lähmende Curare, über das das Physostigmin Sieger bleibt. Nach den Versuchen von $\mathrm{Pal}^{1}$ ) and von Rothberger ${ }^{2}$ ) stellt das Physostigmin die Thätigkeit des durch Curare inactiv gemachten

1) Pal, Centralblatt f. Physiologie. Bd. 14. 1900. S. 255.

2) Rothberger, Pflüger's Archiv. Bd. 87. 1901. S. 11 . 
Muskels wieder her, namentlich am Zwerchfell (Athembewegung). Im Reagenzglase neutralisiren sich die beiden Gifte aber keineswegs. Es bleibt demnach auch hier die gleiche Alternative: entweder das Physostigmin wirkt auf die Muskelfaser selbst oder das lähmende Gift wird durch das erregende gewissermaassen aus dem Nervenendapparat verdrängt, falls man nicht annehmen will, dass der Endapparat zwar in Folge der Curarewirkung nicht mehr leitete, wohl aber für Physostigmin noch erregbar blieb, was ziemlich auf das Gleiche herauskommen würde. Endlich könnte man auch hier die wenig wahrscheinliche Annahme machen, dass der allerperipherste Theil der Nervenendausbreitung zwar durch das erregende, nicht aber durch das lähmende Gift beeinflusst wird.

Etwas complicirter, wenngleich im Wesentlichen analog, liegen die Verhältnisse am Herzen, wenigstens so weit zunächst das Froschherz in Frage kommt. Hier kommt freilich, worauf ich schon unlängst hingewiesen habe ${ }^{1}$, zuvörderst Alles darauf an, ob man der modernen rein myogenen Theorie der Herzfunction huldigt. Thut man das, so bleibt überhaupt keine andere Annahme, als dass das Physostigmin auf den Herzmuskel selbst einwirkt, und zwar vor Allem auf die Automatie crzeugenden Sinusfasern (oder richtiger: Muskelzellen). Daraus würde sich dann wohl auch erklären, warum Schweder²) (unter Kobert's Leitung) den an der Atrioventricularfläche abgeschnürten Ventrikel durch Physostigmin nicht zum Schlagen bringen konnte, falls etwa die Abschnürung so geschah, dass die Sinusfasern oberhalb derselben blieben. Indes neige ich mich, und $z w a r$ gerade auf Grund der einschlägigen pharmakologischen Thatsachen, der myogenen Herztheorie nicht zu und bleibe bei der älteren Annahme der automatischen oder muskulomotorischen Centren (Ganglien) des Froschherzens stehen. Das Physostigmin hebt nun am Froschherzen zunächst den diastolischen Jodalstillstand $\mathrm{auf}^{3}{ }^{3}$. Dieser letztere lässt sich aber auf Grund der älteren neurogenen Theorie nur aus einer Lähmung der automatischen Centren des Froschherzens erklären. Es bleibt also auch hier das erregende Gift zunächst Sieger über das lähmende, und die Alternative ist die gleiche wie oben: entweder das Physostigmin reizt muskuläre Theile des Herzens direct, oder das lähmende Gift wird aus dem Nervenapparat durch das erregende gewissermaassen verdrängt, eliminirt, und die Function der automatischen Centren zeitweilig wieder restituirt. Aber auch das durch Kupfordoppelsalze nahezu zum Stillstand in halber schlaffer Diastole gebrachte Froschherz wird durch Physostigmin wieder zum Schlagen gebracht ${ }^{4}$ ). Dass durch Kupfer der Herzmuskel selbst (nach vorgängiger Reizung) gelähmt wird, so dass er schliesslich auch auf directen fara-

1) Vgl. Harnack, Archiv f. Anat. u. Physiol. Physiol. Abtheil. 1904. S. 415.

2) Schweder, Ueber Eserin etc. Diss. Dorpat 1889.

3) Vgl. Harnack und Witkowski, Archiv f. exper. Path. u. Pharmakol. Bd. XI. S. 1. 1879.

4) Vgl. Harnack und Hafemann, Archiy f. exper. Path. u. Pharmakol. Bd. 17. S. 145 u. Tafel IV, Fig. Vd u. e. 1883. 
dischen Reiz nicht mehr reagirt, davon kann man sich, wie überhaupt am Froschmuskel, leicht überzeugen. Dic Frage, ob das Physostigmin seine Wirkung durch Reizung der Herzcentren oder des Herzmuskels zu Stande bringt, mag hier offen bleiben.

An dem den Herzen der Skorpionen ähnlich gebauten Herzen des Molukkenkrebses (Limulus) hat neuerdings Carlson Versuche angestellt und daraus geschlossen, das Physostigmin wirke primär erregend nur auf den Ganglienapparat, nicht auf die muskulären Theile des Herzens ein. Von einem wirbellosen Thier ist freilich eine directe Uebertragung auf das Froschherz ebenso unthunlich wie von diesem auf das Warmblüterherz.

Die küralich von Winterberg angestellten Versuche beschränken sich auf das Säugethierherz, und hier beobachtet der Autor vor Allem eine Steigerung der Lrregbarkeit des kardialen Hemmungsapparates durch das Physostigmin, eine Wirkung, die von Witkowski und mir seiner Zeit nicht constatirt worden war. Indem er letzteres an sich mit Recht betont, beachtet der Autor vielleicht zu wenig: erstens, dass man seit dreissig Jahren über die Wirkung der Vagusreizung auf das Herz manches zugelernt hat, was damals noch nicht so bekannt war, und sodann, dass für uns seiner Zeit das Froschherz das wiehtigere Object war, wir aber hier vor Allem zu beweisen hatten, dass das Physostigmin dic Vagusendigungen nicht lähmt. Will man wissen, ob es sie erregt, so muss man natürlich vor Allem nachweisen, dass es sie nicht lähmt. Die Meinung aber, das Physostigmin lähme den Hemmungsapparat im Froschherzen, war von Forschern, die vor uns gearbeitet hatten, mehrfach ausgesprochen worden und schien auch nicht so fernliegend. Einmal hebt das Physostigmin den Muskarinstillstand auf, scheint also darin dem Atropin gleich zu wirken, was andererseits gar nicht der Fall ist, indem es den Herzschlag verlangsamt und verstärkt, und sodann wird in der Physostigminwirkung die faradische Reizung des Froschvagus sehr bald erfolglos. Unsere Aufgabe war es nun, zu erweisen, dass das Physostigmin sich so verhält, nicht weil es die Vagusendigungen lähmt, sondern weil es den Herzmuskel — sei es nun direct oder durch Vermittelung der automatischen Ganglien - erregt. Diesen Beweis suchten wir zunächst durch den Versuch: Muskarin-PhysostigminKupfer-Atropin zu führen. Wenn Winterberg Bedenken gegen diesen Versuch hat, so theile ich dieselben insofern, als auch ich den Beweis auf diesen Versuch allein nicht stützen möchte. Es werden dabei vier Herzgifte nach einander zur Wirkung gebracht und es kommt ungemein auf die richtige Dosirung ete. dabei an. Ich habe mich aber keineswegs auf diesen Versuch beschränkt; schon mit Witkowski (l. c. S. 422) stellte ich Versuche am Coats'schen Herzvaguspräparate an und später habe ich (mit Hafemann, l. c.) sehr mühsame Versuche mit dem gleichen Präparate in Combination mit dem Froschherzapparate von Williams ausgeführt. Hierbei ergab es sich, dass, sowie man das Physostigmin derart mit dem herzlähmenden Kupfer combinirt, dass das Herz nicht mehr abnorm kräftig schlägt, die faradische Reizung des Froschvagus wicder den schönsten Effect ergiebt (Stillstand von $1 / 4$ Minute, 
cf. Harnack und Hafemann, l. c. Tafel IV, Fig. Va-o). Der Vagus wird also weder vom Physostigmin noch vom Kupfer gelähmt; letzteres war nämlich auch unrichtiger Weise, und zwar von Luchsinger, behauptet worden. Ebenso wenig aber wird beim Frosch der Vagus durch Physostigmin erregt; denn die künstliche Reizung des Vagusstammes wird beim Frosch in der Physostigminwirkung erfolglos, beim Säugethier aber nicht, wie wir sehr bald feststellen konnten. Es verhält sich hier also ähnlich wie mit dem Digitalin: die Pulsverlangsamung durch Digitalisgifte beruht beim Säugethier sicher auf Vagusreizung (reflectorisch?), beim Frosche aber nicht.

Für das Physostigmin haben nun die Versuche von Winterbery eine Erregung der Vagusendigungen im Säugethierherzen orgeben, und zwar augenscheinlich eine ganz directe. Eine solche Wirkung haben seiner Zeit Witkowski und ich nicht constatirt. Wenn Winterberg meint, wir hätten sie übersehen, weil wir unseren Versuchsthieren durch starke Curarisirung die Vagi gelähmt hätten, so irrt er doch und übersieht dabei erstens, dass wir am Hunde trotz starker Curarisirung die faradische Vagusreizung erfolgreich gefunden haben (H. u. W., l. c. S. 434) und zweitens, dass wir (S. 433) ausdrücklich angeben, das Physostigmin verlangsame den Puls, auch nachdem durch Atropin oder Curare der Einfluss der Hemmungsnerven völlig ausgeschaltet worden. Es war uns also die Möglichkeit der Vaguslähmung durch Curare nicht nur wohl bekannt, sondern wir haben auch mit voller Absicht die Wirkung bis zu dieser Intensität gesteigert. Die Pulsverlangsamung durch Physostigmin trotz vollständiger Lähmung der Vagi durch Atropin oder Curare hat ja auch Winterberg (Punkt 4 seiner Conclusionen) bestätigt.

Warum haben wir aber die Vaguserregung durch Physostigmin beim Warmblüter nicht beobachtet? Wenn es nicht doch eine Verschiedenheit der Präparate war, so ist zunächst darauf hinzuweisen, dass man jetzt über den Einfluss einer starken Vagusreizung auf das Herz etwas mehr weiss als vor über dreissig Jahren. Es könnten aber noch andere Gründe hinzukommen. Einmal hatten wir auch am Warmblüterherz zunächst zu beweisen, dass das Physostigmin die Vagi nicht lähmt. Das liess sich freilich hier sofort und sehr leicht darthun, da die Vagusreizung in der Physostigminwirkung nicht, wie beim Frosch, erfolglos wird.

Sodann beobachteten wir bei unseren Blutdruckversuchen hauptsächlich zwei Momente der Physostigminwirkung: das Herz schlägt langsamer und kräftiger und der Blutdruck kann, wenigstens zeitweilig, erhöht werden. Die erstere Erscheinung liesse sich natürlich mit eincr Vagusreizung in Einklang bringen, die letztere aber nicht, und da das Physostigmin, was Winterberg ja bestätigt hat, den Puls auch am atropinisirten Herzen verlangsamte, so konnten wir nach der damaligen allgemeinen Voraussetzung nicht schliessen, dass die Verlangsamung auf einer Vagusreizung beruhe. Die Verhältnisse des Blutdrucks gestalten sich in der Physostigminwirkung complicirter, weil Einflüsse vom centralen Nervensystem aus durch die Vasomotoren etc. hinzukommen können, so dass grössere Dosen den Blutdruck nachträglich zu erniedrigen scheinen. 
Winterberg schliesst übrigens (Punkt 7 seiner Conclusionen), dass das Physostigmin wahrseheinlich auch eine ndirecte Reizwirkung auf das Herz" besitze, und ich glaube ihm darin beistimmen zu müssen. Das würde sich übrigens meines Erachtens am besten am isolirten Warmblüterherzen entscheiden lassen. Therapeutisch wäre wohl das Physostigmin nicht nur bei tachykardischen Anfällen, die auf primärer Herabsetzung des Vagustonus beruhen, sondern wahrscheinlich auch als cin Herzanalepticum brauchbar, wenn es eben nicht durch die schlimmen Wirkungen vom Centralnervensystem her so bald gefährlich würde. Durch Morphin lassen sich diese Wirkungen zum Theil beseitigen, soweit sie erregende sind, aber sie können leider rasch zu lähmenden werden.

Zur Entscheidung der wichtigen principiellen Frage, von der ich oben ausgegangen bin, ist aber von erheblicher Bedeutung der .Punkt 8 der Conclusionen Winterberg's: darnach wird nämlich innerhalb gewisser Grenzen nicht nur die Atropin- und Curare-, sondern auch die Nikotinlähmung des Vagus durch Physostigmin aufgehoben. Hier kann es sich doch nur um den nämlichen Nervenapparat handeln und das Physostigmin bleibt also auch hier Sieger über die lähmenden Gifte, genau wie an den willkürlichen Muskeln gegenüber dem Curare. Damit wäre die frühere principielle Auffassung, dass solches nicht möglich sei, widerlegt und damit fiele zugleich auch der Zwang, die Physostigminwirkung als directe Muskelwirkung zu deuten, weg. Auffallend bleibt indes, dass sowohl $\mathrm{Pal}$ wie Rothberger betonen, das Physostigmin sei ein directer Antagonist des Curares, aber nicht des Atropins, während Witkowski und ich und viele anderen Beobachter eher das Umgekehrte beobachteten. Da ist unzweifelhaft noch ein gewisser Widerspruch im Thatsächlichen vorhanden und das bringt mich immer wieder auf Zweifel an der vollen Identität der Präparate, mag aber vielleicht auch an gewissen Verschiedenheiten der Versuchsbedingungen liegen.

Wie dem aber auch sein mag, so viel scheint doch zweifellos festgestellt zu sein, dass das Physostigmin bei bestimmiten Combinationen dem den gleichen Nervenapparat lähmenden Gifte gegenüber Siegor bleibt. Damit gewinnt die Physostigminwirkung überhaupt ein besonderes Interesse, Man kann vielleicht nicht sagen, dass es in diesem seinem Verhalten einzig dasteht, aber der Fall ist doch ein verhältnissmässig seltener, und andere den Nervenapparat erregende Gifte, wie Muskarin ete., vermögen es eben nicht. Warum verhält sich gerade das Physostigmin so? Hier wäre in der That die Beantwortung der Frage nach dem Warum? von grösster Bedeutung.

Mit der thatsächlichen Anerkennung eines solchen Verhaltens des Physostigmins fällt aber auch der Zwang zur Annahme einer directen Muskelwirkung weg und fällt auch die früher allgemein acceptirte principielle Auffassung, von der ich bei meinen Darlegungen ausgegangen bin. Berücksichtigt man das antagonistische Verhalten im Einzelnen, so gewinnt man in der That den Eindruck, als ob das erregende und das lähmende Gift sich um den Nervenapparat, auf den sie einwirken, 
stritten, und dass es abgesehen von den Dosirungsverhältnissen auch von sonstigen Umständen abhängt, wer zeitwcilig Sieger bleibt. Rothbergor beobachtete, dass der durch Curare inactiv gemachte Muskel durch Physostigmin wieder zur Function gebracht wird, am leichtesten das Zwerchfell, aber auch alle sonstigen willkürlichen Muskeln, die dann durch erneute Curarisirung wieder inactiv werden. Das zuletzt zugeführte Gift erringt also einen gewissen Vorrang, durchaus analog dem, was ich in Betreff des Verhältnisses: Physostigmin zu $\Lambda$ tropin an der Iris beobachtet habe. Giebt man das erregende und lähmende Mittel zugleich gemengt, so überwiegt erheblich das letztere. Die beiden Gifte wirken aber auf einander - im Reagenzglase - chemisch gar nicht cin und es ist höchst unwahrscheinlich, dass sie in dem lebenden Organe direct auf einander einwirken. Man kann nur annehmen, dass das zuletzt zugeführte das erste verdrängt, und war das erste das lähmende, so kann eben dic verloren gegangene Function wieder restituirt werden.

So hat uns die ungemein interessante Wirkung des Physostigmins auf muskuläre Organe werthvolle Aufschlüsse gegeben und eine nicht unerhebliche Modificirung einer principiell-pharmakologischen Auffassung veranlasst. Immerhin muss man zugeben, dass der Beweis, dass das Physostigmin nicht auch auf muskuläre Elemente selbst einwirkt, bisher noch nicht strict hat geführt werden können: ich vermag denselben auch nicht mit Sicherheit aus den Versuchen von Rothberger zu entnehmen, obschon er selbst den Schluss zieht, dass Curare und Physostigmin auf den nämlichen Angriffspunkt einwirken, was dann natürlich nur der motorische Nervenendapparat sein könnte. 